Michael Leinert

\title{
On a theorem of Wiener
}

\author{
Received: 12 September 2000
}

\begin{abstract}
Wiener has shown that an integrable function on the circle $T$ which is square integrable near the identity and has nonnegative Fourier transform, is square integrable on all of $T$. In the last 30 years this has been extended by the work of various authors step by step. The latest result states that, in a suitable reformulation, Wiener's theorem with " $p$-integrable" in place of "square integrable" holds for all even $p$ and fails for all other $p \in(1, \infty)$ in the case of a general locally compact abelian group. We extend this to all IN-groups (locally compact groups with at least one invariant compact neighbourhood) and show that an extension to all locally compact groups is not possible: Wiener's theorem fails for all $p<\infty$ in the case of the $a x+b$-group.
\end{abstract}

The following theorem is attributed to N. Wiener: If $f$ is a Lebesgue integrable function on the circle group $T$ with nonnegative Fourier coefficients, then square integrability of $f$ in some neighbourhood of the group identity implies square integrability on all of $T$. It follows from this, that the same result with " $p$-integrability" instead of "square integrability" still holds, if $p$ is an even natural number. For noneven $p \in(1, \infty)$ however, the theorem fails, as was shown by Wainer [Wa] $(p<2)$ and Shapiro [Sh] ( $p>2, p$ not even) in 1969 and 1975, respectively. The reason for the failure of Wiener's theorem for noneven $p$ lies in the failure of the upper majorant property for $L^{p}(T)$ for such $p$. Bachelis [Ba] in 1973 showed that for infinite compact abelian $G$ the upper majorant property for $L^{p}(G)$ fails for noneven $p \in[1, \infty)$ except for the case where $\hat{G}$ is a torsion group and $p \in(2, \infty)$ is noneven. This gap was closed by Fournier [Fo 1] 1974 thus opening the way for a generalization of the circle results to compact abelian groups, which was done by M. Rains [Ra] in 1985. Wiener's theorem for the real line (replace $T$ above by $R$ ) was disproved by Kawazoe, Onoe, and Tachizawa [Ka On Ta] in 1994. However, Fournier reformulated Wiener's theorem equivalently and was able to extend its validity/nonvalidity for $p$ even / $p$ noneven in its new form to all locally compact abelian groups. We further extend this result to all IN-groups in Theorem 1.6 and construct a counterexample which shows that Wiener's theorem fails for all $p$ on the $a x+b$-group (Theorem 1.16).

$G$ always denotes a locally compact group, $e$ its group identity, $\lambda$ a left Haar measure on $G$. For $\int f d \lambda$ we also write $\int f(x) d x$ or just $\int f$. The subsets of

M. Leinert: Ruprecht-Karls-Universität, Institut für Angewandte Mathematik, Im Neuenheimer Feld 294, 69120 Heidelberg, Germany. e-mail: leinert@math.uni-heidelberg.de

Mathematics Subject Classification (2000): 43A35 
$G$ which we consider are always assumed to be $\lambda$-measurable. For $A \subset G,|A|$ denotes $\lambda(A), \bar{A}$ (resp. $\AA$ ) the closure (resp. interior) of $A$. Note that for $\lambda$-measurable $f: G \rightarrow[0, \infty)$ the measure $f \lambda$ is not necessarily outer regular (even for $G=R$ ). The modular function of $G$ is denoted by $\Delta$, the space of all complex valued continuous functions by $C(G)$, the subspace of functions in $C(G)$ with compact support by $K(G)$. For functions $f, g$ on $G$ and elements $x, y \in G$ we set $\langle f, g\rangle=\int f g,(y f)(x)=f(y x),\left(f_{y}\right)(x)=f(x y), \bar{f}(x)=\overline{f(x)}$ (complex conjugation), $\check{f}(x)=f\left(x^{-1}\right), \tilde{f}(x)=\overline{f\left(x^{-1}\right)}, f^{*}(x)=\Delta(x)^{-1} \overline{f\left(x^{-1}\right)}, f * g$ the convolution of $f$ and $g$. If $f$ is locally integrable and satisfies $\left\langle f, g^{*} * g\right\rangle \geq 0$ for all $g \in K(G), f$ is called positive definite. For continuous $f$, this coincides with the usual definition of positive definiteness (see [Di] p. 287 and p. 296). If $U \subset G$ satisfies $x U x^{-1} \subset U$ for all $x \in G, U$ is called invariant. For $U \subset G, \chi_{U}$ denotes its characteristic function. For $p, q \geq 1$, the amalgam space $\left(L^{p}, 1^{q}\right)$ can be defined in several (equivalent) ways, for instance as the space of measurable functions $f$ on $G$ with $x \mapsto\left\|f \chi_{x} U\right\|_{p}$ in $L^{q}$ (where $U$ is a relatively compact neighbourhood of $e$ ). Using a different neighbourhood $U^{\prime}$ leads to the same space with an equivalent norm. If $\pi$ is a continuous unitary representation of $G$ on a Hilbert space, its canonical extension to $L^{1}(G)$ is denoted by the same letter $\pi$.

The following condition $(*)_{p}$ has already been considered by J.J.F. Fournier:

Definition and Remark 1.1. Let $1 \leq p \leq \infty$ and $G$ be a locally compact group. We say that $G$ has property $(*)_{p}$, if for every relatively compact neighbourhood $U$ of the identity e, there is a constant $C_{U}$ such that for every locally integrable positive definite function $g: G \rightarrow \mathbf{C}$ and every $x \in G$ we have

$$
\left\|g \chi_{x U}\right\|_{p} \leq C_{U}\left\|g \chi_{U}\right\|_{p}
$$

or equivalently (in the case $p<\infty$ )

$$
\int_{x U}|g|^{p} \leq C_{U}^{\prime} \int_{U}|g|^{p}
$$

It is easy to see that if such an inequality hold for one choice of $\mathrm{U}$, it holds for every $U$ (with a possibly different constant, of course).

Remark 1.4. For $1<p \leq \infty$, property $(*)_{p}$ clearly implies that for locally integrable positive definite functions $g$ on $G$ the following holds:

(i) $)_{p}$ If $\left\|g \chi_{U}\right\|_{p}<\infty$ for some relatively compact neighbourhood $U$ of the identity $e$, then $g \in\left(L^{p}, 1^{\infty}\right)$.

Conversely, $(i)_{p}$ implies $(*)_{p}$ if $G$ is abelian. For discrete $G$ this follows from 1.5 (a) below. Now suppose $G$ is nondiscrete abelian and $(i)_{p}$ holds. If $f \in L^{1}(G)$ with $\hat{f} \geq 0$, then $f$ is positive definite, because for $h \in K(G)$ we have $\left\langle f, h^{*} * h\right\rangle=$ $\int(f * \breve{h}) \cdot \bar{h}=\int \hat{f}|\hat{h}|^{2} \geq 0$. For noneven $p \in(1, \infty)$, by [Fo 2], Theorem 3.9 there is such an $f \in L^{1}(G)$ which is $p$-integrable near the identity but is not in $\left(L^{p}, 1^{\infty}\right)$. So, by $(i)_{p}, p$ must be even. By 1.6 below, $(*)_{p}$ holds. 
Proposition 1.5. (a) If $G$ is discrete, it has property (*) ${ }_{p}$ for every $p>0$. (b) If $G$ is locally compact, it always has property $(*)_{\infty}$.

Proof. (a) For $G$ discrete, $U=\{e\}, x \in G$ and $g$ positive definite we have

$$
\left\|g \chi_{x U}\right\|_{p}=|g(x)| \leq g(e)=\left\|g \chi_{U}\right\|_{p} .
$$

(b) For $g$ continuous positive definite we have

$$
\left\|g \chi_{x} U\right\|_{\infty} \leq\|g\|_{\infty} \leq g(e)=\left\|g \chi_{U}\right\|_{\infty} .
$$

For $g$ locally integrable positive definite with $\left\|g \chi_{U}\right\|_{\infty}<\infty$ let $\mathrm{V}=\mathrm{V}^{-1}$ be a neighbourhood of $e$ with $\mathrm{V}^{2} \subset U$ and set $h=\left(\frac{1}{|V|} \chi_{V}\right) * g *\left(\frac{1}{|V|} \chi_{V}\right)^{2}$ which is continuous positive definite. For $k \in K(G)$ we have $|\langle h, k\rangle| \leq\|h\|_{\infty}\|k\|_{1}=$ $h(e)\|k\|_{1} \leq\left\langle g,\left(\frac{1}{|V|} \chi_{V}\right)^{*} *\left(\frac{1}{|V|} \chi_{V}\right)\right\rangle\|k\|_{1} \leq\left\|g \chi_{U}\right\|_{\infty} \cdot 1 \cdot\|k\|_{1}$. Since $\langle h, k\rangle \rightarrow$ $\langle g, k\rangle$ for $V \rightarrow\{e\}$, it follows $|\langle g, k\rangle| \leq\left\|g \chi_{U}\right\|_{\infty}\|k\|_{1}$, which implies $\|g\|_{\infty} \leq$ $\left\|g \chi_{U}\right\|_{\infty}$. In particular we have $\left\|g \chi_{x} U\right\|_{\infty} \leq\left\|g \chi_{U}\right\|_{\infty}$. So property $(*)_{\infty}$ holds and we see that for $p=\infty$ the constant $C_{U}$ can always be chosen to be one.

Theorem 1.6. Let $G$ be a nondiscrete IN-group (i.e. having at least one invariant relatively compact e-neighbourhood). Let $1<p \leq \infty$. Then $G$ has property $(*)_{p}$ if and only if $p$ is an even integer or $p=\infty$.

The proof of this theorem will follow from a series of Lemmas and Propositions. For $p=\infty$, the assertion follows from 1.5(b).

The general line of proof is as follows: for the "if" part, we reduce the problem to continuous functions where it is easy to obtain the desired result. For the "only if" part, we reduce to a situation where it is possible to lift a commutative counterexample.

Lemma 1.7. Let $1 \leq p<\infty$. Let $G$ be locally compact, $f: G \rightarrow C$ locally integrable and $W \subset G$ relatively compact (or with finite Haar measure $|W|$ ).

(i) Let $Z$ be a symmetric neighbourhood of the identity e such that $\int_{Z W}|f|^{p}<$ $\infty$ and let $V \subset Z$ be relatively compact with $\left\|f \chi_{Z W}-{ }_{y^{-1}}\left(f \chi_{Z W}\right)\right\|_{p}<\varepsilon$ for all $y \in V$. If $h \in L^{1}(G)^{+}$has $\int h=1$ and vanishes outside $V$, then $\| \chi_{W}(f-$ $h * f) \|_{p}<\varepsilon$.

(ii) If $W$ is open, $\int_{W}|f|^{p}=\infty$, and for every $V$ from a basis of neighbourhoods of $e, h_{V}$ is a function in $L^{1}(G)^{+}$with $\int h_{V}=1$ and vanishing outside $V$, the net $\int_{W}\left|h_{V} * f\right|^{p}$ converges to infinity as $V \rightarrow\{e\}$.

Proof. (i) As $h * f(x)=\int h(y) f\left(y^{-1} x\right) d y$ and $h=0$ outside $\mathrm{V}$, the values of $h * f(x)$ for $x \in W$ depend only on the values of $f$ on $V^{-1} W \subset Z W$. So $\left\|\chi_{W}(f-h * f)\right\|_{p}=\left\|\chi_{W}\left(f \chi_{Z W}-h *\left(f \chi_{Z W}\right)\right)\right\|_{p} \leq\left\|f \chi_{Z W}-h *\left(f \chi_{Z W}\right)\right\|_{p}$, which is $<\varepsilon$ by the assumptions on $V$ and $h$.

(ii) It suffices to prove the assertion for a subnet. As $f$ is locally integrable and $V$ may be assumed to be relatively compact, the set of functions $\left(h_{V} * f\right) \cdot \chi_{W}$ is bounded in $L^{1}(G)$. If it were also bounded in $L^{p}(G)$, there would be a weak* convergent subnet $\left(h_{V^{\prime}} * f\right) \chi_{W} \rightarrow F \in L^{p}(G)$. In particular $\left\langle h_{V^{\prime}} * f, g\right\rangle \rightarrow\langle F, g\rangle$ for all $g \in K(G)$ with support in $W$. But $\left\langle h_{V^{\prime}} * f, g\right\rangle=\left\langle f, h_{V^{\prime}}^{*} * g\right\rangle \rightarrow\langle f, g\rangle$, so $f=F$ as Radon measures on $W$, hence $f=F$ almost everywhere on $W$. This implies $f \chi_{W} \in L^{p}(G)$ in contradiction to the assumption. 
Remark 1.8. The Lemma holds for twosided convolution, too:

(a) In (i) assume $\int_{Z W Z}|f|^{p}<\infty$ and $V \subset Z$ relatively compact such that $\left\|f \chi_{Z W Z}{ }_{y^{-1}}\left(f \chi_{Z W Z}\right)\right\|_{p}<\varepsilon$ and $\left\|f \chi_{Z W Z}-\left(f \chi_{Z W Z Z}\right)_{y}\right\|_{p}<\varepsilon$ for all $y \in V$. The conclusion then is $\left\|\chi_{W}(f-h * f * \tilde{h})\right\|_{p}<\varepsilon$.

(b) In (ii) we obtain (without any change of assumptions)

$$
\int_{W}\left|h_{V} * f * \tilde{h}_{V}\right|^{p} \rightarrow \infty \quad \text { as } V \rightarrow\{e\} .
$$

The absence of the modular function $\Delta$ does not disturb the proof, as for $V$ sufficiently small $\Delta$ is uniformly on $V$ as near to 1 as we please.

Proposition 1.9. Let $1 \leq p<\infty, G$ locally compact, $W \subset G$ open relatively compact, $x \in G, C>0$ and $f$ locally integrable and positive definite with $\int_{Z W Z}|f|^{p}<\infty$ for some e-neighbourhood $Z$ and

$$
\int_{x W}|f|^{p}>C \int_{W}|f|^{p}
$$

Suppose $\int_{x W}|f|^{p}=\infty$ or $\int_{Z^{\prime} x W Z^{\prime}}|f|^{p}<\infty$ for some e-neighbourhood $Z^{\prime}$.

Then there is g continuous and positive definite such that (i) holds with $g$ instead of $f$.

Proof. For any relatively compact $e$-neighbourhood $V \subset Z$ let $h_{V}=\frac{1}{|V|} \chi_{V}$ and $g_{V}=h_{V} * f * \tilde{h}_{V}$. If $\int_{x W}|f|^{p}=\infty$, (resp. $\int_{Z^{\prime} x W Z^{\prime}}|f|^{p}<\infty$ ) we have by (a) and (b) of the Remark (resp. by applying (a) twice) $\int_{W}\left|g_{V}\right|^{p} \rightarrow \int_{W}|f|^{p}$ and $\int_{x W}\left|g_{V}\right|^{p} \rightarrow \int_{x W}|f|^{p}$ as $V \rightarrow\{e\}$. So for $V$ small enough, $g_{V}$ will satisfy (i). Clearly $g_{V}$ is continuous. For $r \in K(G)$ we have $\left\langle g_{V}, r^{*} * r\right\rangle=\left\langle f, h_{V}^{*} * r^{*} *\right.$ $\left.r * h_{V}\right\rangle \geq 0$, so $g_{V}$ is positive definite. Thus, for $V$ small enough, $g=g_{V}$ satisfies the assertion.

Remark 1.10. If $G$ is amenable, the function $g$ in the Proposition can be chosen to have compact support, in addition. This is achieved by multiplying the original $g$ with a continuous positive definite function in $K(G)$ which is near to one on a compact set containing $W$ and $x W$.

Proposition 1.11. Let $1 \leq p<\infty$ and $G$ be locally compact. Then $G$ has property $(*)_{p}$ if and only if (1.2) is satisfied with continuous positive definite functions.

Proof. Suppose that $(*)_{p}$ does not hold. Then there is an open relatively compact neighbourhood $W$ of $e$ such that for any constant $C>0$ there is $x \in G$ and a locally integrable positive definite function $f$ such that (i) in the last Proposition holds. In particular $\int_{W}|f|^{p}$ is finite.

(a) Suppose first there are $W, f$ and $x$ such that $\int_{x W}|f|^{p}=\infty$. We then may assume $\int_{Z W Z}|f|^{p}<\infty$ for some $e$-neighbourhood $Z$ (otherwise replace $W$ by an $e$-neighbourhood $Y$ with $Y^{3} \subset W$. Since finitely many translates of $Y$ cover $W$, there must be some $x^{\prime} \in G$ with $\left.\int_{x^{\prime} Y}|f|^{p}=\infty\right)$. Applying the last Proposition we see that we can satisfy (i) there with a continuous positive definite function $g$ for every $C>0$. 
(b) When case (a) does not apply, $f$ is locally $p$-integrable, so we have $\int_{Z W Z}|f|^{p}$ $<\infty$ and $\int_{Z x W Z}|f|^{p}<\infty$ automatically satisfied for every relatively compact $e$-neighbourhood Z. Applying the last Proposition again we obtain that (i) there is satisfied with a continuous positive definite function $g$. As $C$ was arbitrary, (1.2) does not hold with continuous positive definite functions. So the assertion is proved.

If $p$ is even, $p=2 k$ say, and $g$ is continuous positive definite on $G,|g|^{n}=g^{k} \cdot \bar{g}^{k}$ is continuous positive definite and nonnegative, so in order to prove $(*)_{p}$ in this case it suffices to establish the following property (*) for $G$ :

Definition and Remark 1.12. We say that $G$ has property (*) if for every (equivalently: for one) relatively compact neighbourhood $U$ of $e$, there is a constant $C_{U}$ such that

$$
\int_{x U} g \leq C_{U} \int_{U} g
$$

for every $x \in G$ and $g \in C(G)^{+}$which is positive definite.

An equivalent formulation of $(*)$ is:

For every (equivalently: for one) nonzero $h \in K(G)^{+}$which is positive definite, there is a constant $C_{h}$ with

$$
\int x h \cdot g \leq C_{h} \int h g
$$

for all positive definite $g \in C(G)^{+}$and $x \in G$.

The equivalence of formulations follows form the fact that, given $h$, there are constants $\alpha, \beta>0$ and relatively compact $e$-neighbourhoods $U, U^{\prime}$ such that $\alpha \chi_{U} \leq h \leq \beta \chi_{U^{\prime}}$, and given $U$, there are positive definite $h_{1}, h_{2} \in K(G)^{+}$ with $h_{1} \leq \chi_{U} \leq h_{2}$ (for $h_{1}$ take a suitable multiple of $\chi_{V} * \chi_{V-1}$ where $V$ is a relatively compact $e$-neighbourhood with $V V^{-1} \subset U$. For $h_{2}$ take a suitable multiple of $\chi_{V^{\prime}} * \chi_{\left(V^{\prime}\right)^{-1}}$ where $V^{\prime}$ is relatively compact and contains the closure $\bar{U}$ in its interior $\stackrel{\circ}{V^{\prime}}$ )

For IN-groups, property (*) is easy to obtain:

Lemma 1.13. For $G$ locally compact, $x \in G, f \in L^{1}, g$ continuous positive definite on $G$ we have

$$
\left|\int\left(f^{*} * \delta_{x} * f\right) \cdot g\right| \leq \int\left(f^{*} * f\right) \cdot g .
$$

Proof. If $\pi$ is a continuous unitary representation of $G$ on a Hilbert space $H$ and $\xi \in H$ with $g(s)=(\pi(s) \xi \mid \xi)$ for $s \in G$, we have

$$
\begin{aligned}
& \left|\int\left(f^{*} * \delta_{x} * f\right) \cdot g\right|=\left|\left(\pi\left(f^{*} * \delta_{x} * f\right) \xi \mid \xi\right)\right| \\
& \quad=|(\pi(x) \pi(f) \xi \mid \pi(f) \xi)| \leq\|\pi(f) \xi\|^{2}=\left(\pi\left(f^{*} * f\right) \xi \mid \xi\right)=\int\left(f^{*} * f\right) \cdot g .
\end{aligned}
$$


Remark 1.14. If $G$ has an invariant relatively compact neighbourhood $U$ (and hence $G$ is unimodular), letting $f=\chi_{U}$ in the Lemma and using the fact that $\chi_{U} * \delta_{x}=$ $\delta_{x} * \chi_{U}$ and $h=\chi_{U}^{*} * \chi_{U}=\tilde{\chi}_{U} * \chi_{U}$ is positive definite, we obtain that $G$ has property (*) and the best constant $C_{h}$ for this $h=\tilde{\chi}_{U} * \chi_{U}$. is one.

In particular, (*) holds for all abelian and all compact groups.

Thus far we have proved the "if"-part of the Theorem. Suppose now $p \in[1, \infty)$ to be not an even integer. If $G$ is locally compact unimodular having $(*)_{p}$ and $K$ is a compact normal subgroup of $G$, then $G / K$ has $(*)_{p}$ as is easily seen (because a positive definite function on $G / K$ composed with the canonical projection is positive definite on $G$, and the Haar measure $d x$ on $G$ can be written $d k d \dot{x}$ where $d k$ resp. $d \dot{x}$ are Haar measures of $K$ resp. $G / K)$. So to disprove $(*)_{p}$ for $G$, it is sufficient to disprove it for $G / K$ where $K$ is compact normal in $G$. It also suffices to disprove $(*)_{p}$ for an open subgroup of $G$. We proceed by reducing the problem to cases where $(*)_{p}$ is already known to fail or to the case of an infinite compact group of finite topological dimension. Let $G$ be a nondiscrete IN-group. There is a compact normal subgroup $K$ of $G$ such that $\dot{G}=G / K$ is a SIN-group (i.e. having a basis of invariant relatively compact neighbourhoods) (see for instance [Ho Mo], p. 49).

(a) Suppose $\dot{G}$ is discrete. Then $K$ is open in $G$ and nondiscrete. Divide by the identity component $K_{0}$, so $K / K_{0}$ is totally disconnected and compact.

(i) Suppose $K / K_{0}$ is discrete. Then $K_{0}$ is open in $K$, so $K_{0}$ is nondiscrete connected and compact. Now proceed as in (b) with $K_{0}$ in place of $\dot{G}$ there.

(ii) Suppose $K / K_{0}$ is nondiscrete. It is also compact and totally disconnected, hence of topological dimension zero. Dividing out a suitable compact normal subgroup, if necessary, by an application of [He Ro], Theorem 8.7, we obtain an infinite metrizable totally disconnected compact group. (Choose $U_{n}$ there such that $U_{n}^{2} \stackrel{\subset}{\neq} U_{n-1}$ in order to guarantee that the quotient is infinite). This case is treated in the next proposition.

(b) Suppose $\dot{G}$ is nondiscrete. Being a SIN-group, $\dot{G}$ is pro-Lie i.e. it has arbitrarily small compact normal subgroups $H$ such that the quotient $\tilde{G}=\dot{G} / H$ is Lie (see [Gr Mz], p. 11) and is still an IN-group (as we took the quotient of a SIN-group by a normal subgroup contained in an arbitrarily small $e$-neighbourhood).

(i) Suppose $\tilde{G}=\dot{G} / H$ is nondiscrete. Then its identity component $\tilde{G}_{0}$, is a nondiscrete connected Lie group and IN-group. By [Ho Mo], p. 50 the topological commutator group of $\tilde{G}_{0}$, call it $C$, is compact. If the (abelian) group $\tilde{G}_{0} / C$ is nondiscrete, it has not property $(*)_{p}$ by [Fo 2], Theorem 3.9. If $\tilde{G}_{0} / C$ is discrete, $C$ is open in $\tilde{G}_{0}$, so $C=\tilde{G}_{0}$, hence $C$ is a nondiscrete compact connected Lie group. This case is treated in the next proposition.

(ii) If $\dot{G} / H$ is discrete, $H$ is open in $\dot{G}$, hence nondiscrete, and compact. Consider $H / \dot{G}_{0}$ where $\dot{G}_{0}$ is the identity component of $\dot{G}$. If this quotient is nondiscrete, it is totally disconnected and compact. We may assume it to be metrizable (see (a)(ii) above), so this case is treated in the next proposition. If the quotient is discrete, $\dot{G}_{0}$ is open in $\dot{G}$, hence a nondiscrete connected compact group. If we now repeat the first step in (b), dividing out a compact normal subgroup $H_{1}$ that is strictly 
contained in $\dot{G}_{0}$, the quotient $\dot{G}_{0} / H_{1}$ must be nondiscrete compact Lie, and this case is treated in the next proposition.

Proposition 1.15. Let $1<p<\infty$, $p$ not even. Let $G$ be an infinite metrizable compact group of finite topological dimension. There is a neighbourhood $U$ of the identity such that for every $C>0$ there is $x \in G$ and $f \in C(G)^{+}$positive definite with $\left\|\left.f\right|_{x U}\right\|_{p}>C\left\|\left.f\right|_{U}\right\|_{p}$. So $G$ does not have property $(*)_{p}$.

Proof. By Zelmanov's theorem (see [Ze]) there is an infinite abelian subgroup A. We may assume it to be closed (otherwise take its closure) and hence nondiscrete. By [Ra] or [Fo 2], there is $g \in L^{1}(\mathrm{~A})$ positive definite, a neighbourhood $W$ of $e$ in $A$ and $x \in A$ such that $\int_{W}|g|^{p}<\infty$ but $\int_{x W}|g|^{p}=\infty$. Note that $W$ can be chosen arbitrarily small (if $W^{\prime} \subset W$ is open, we can cover $x W$ with finitely many translates of $W^{\prime}$ and so find a translate $s W^{\prime}$ with $\int_{s W^{\prime}}|g|^{p}=\infty$ ). So we may assume that for some $e$-neighbourhood $Z=Z^{-1}$ in $A$ we have $\int_{Z W}|g|^{p}<\infty$. As $G$ has finite topological dimension, there is (see, [Mo] p. 646/47) a neighbourhood $Q$ of $\dot{e}=A e$ in $\dot{G}=G / A$ and a continuous map $\sigma: Q \rightarrow G$ with $\sigma(\dot{e})=e$ and $A \sigma(\dot{y})=\dot{y}$ for all $\dot{y} \in Q$. Choose an $e$-neighbourhood $Z_{0}=Z_{0}^{-1}$ in $A$ such that $Z_{0}^{2} \subset Z$ and $\left\|g \chi_{Z W}{ }_{y^{-1}}\left(g \chi_{Z W}\right)\right\|_{p}<\varepsilon$ for all $y \in Z_{0}$.

Denote the set $\left\{a \sigma(q) \mid a \in Z_{0}, q \in Q\right\}$ by $Z_{0} \times Q$ (which is topologically justified). Let $V=V^{-1}$ be an invariant $e$-neighbourhood in $G$ such that $V^{2}$ is contained in $Z_{0} \times Q$. Let $h=\frac{1}{|V|^{2}} \chi_{V} * \chi_{V}$. Now $g * h$ (convolution of measures on $G$ ) is an $L^{1}$-function on $G$ given by

$$
\begin{aligned}
g * h(s) & =\int_{A} g(y) h\left(y^{-1} s\right) d y \\
& = \begin{cases}\left(\left.g * h_{\sigma(\dot{s})}\right|_{A}\right)(a) & \text { where } s=a \sigma(\dot{s}), \dot{s} \in Q \\
0 & \text { for } \dot{s} \notin Q .\end{cases}
\end{aligned}
$$

Denote $\left.h_{\sigma(\dot{s})}\right|_{A}$ by $h_{\dot{s}}$. We have $\left\{a \in A \mid h_{\dot{s}}(a) \neq 0\right\} \subset Z_{0}$, So

$$
\begin{gathered}
\int_{Z_{0} W}\left|g * h_{\dot{s}}\right|^{p} \leq\left(\int h_{\dot{s}}\right)^{p} \int_{Z_{0}^{2} W}|g|^{p} \quad \text { and hence } \\
\int_{Z_{0} W \times Q}|g * h|^{p} \leq \int_{Z_{0}^{2} W}|g|^{p} \cdot \int\left(\int h_{\dot{s}}\right)^{p} d v(\dot{s})
\end{gathered}
$$

where $v$ is a measure on $\dot{G}$ satisfying

$$
\int_{G} f(x) d x=\int_{\dot{G}} \int_{A} f(\xi x) d \xi d v(\dot{x}) \text { for } f \in L^{1}(G) \text {. }
$$

Now choose $C>0$ arbitrary large and according to (ii) of Lemma 1.7 choose an e-neighbourhood $Z_{1}=Z_{1}^{-1} \subset Z_{0}$ in $A$ such that $\left.\left.\int_{Z_{0} W}\right|_{x} g * k\right|^{p}>C^{p}$ for all 
$k \in\left(L^{1}(A)\right)^{+}$with $\int k=1$ and vanishing outside $Z_{1}$. Let $V$ as above even satisfy $V^{2} \subset Z_{1} \times Q$. Then we have $\left\{a \in A \mid h_{\dot{s}}(a) \neq 0\right\} \subset Z_{1}$ and hence

$$
\int_{Z_{0} W}\left|x g * h_{\dot{s}}\right|^{p} \geq C^{p} \cdot\left(\int h_{\dot{s}}\right)^{p} \text { for } \dot{s} \in Q,
$$

So

$$
\int_{Z_{0} W \times Q}|x g * h|^{p} \geq C^{p} \int\left(\int h_{\dot{s}}\right)^{p} d v(\dot{s}) .
$$

So letting $U=Z_{0} W \times Q$ and $f=g * h$ we get integrable (and in fact continuous bounded) functions such that $\left\|\left.f\right|_{U}\right\|_{p}$ and $\left\|\left.f\right|_{x U}\right\|_{p}$ differ by an arbitrarily large factor (in fact going to $\infty$ ) as $V \rightarrow\{e\}$.

As $g * h=\frac{1}{|V|} \chi_{V} * g * \frac{1}{|V|} \chi_{V}$ (recall that $V$ is $G$-invariant), for $r \in K(G)$ we have $\left\langle g * h, r^{*} * r\right\rangle=\left\langle g,\left(\frac{1}{|V|} \chi_{V}\right)^{*} * r^{*} * r *\left(\frac{1}{|V|} \chi_{V}\right)\right\rangle$.

The convolution product to the right is positive definite (because $G$ is unimodular) and so is its restriction to $A$. The latter belongs to the Fourier-Algebra of $A$ and hence can be written $d * \tilde{d}$ with $d \in L^{2}(A)$. As $\tilde{d}=d^{*}$ ( $A$ is unimodular) and choosing $d_{n} \in K(A)$ with $\left\|d-d_{n}\right\|_{2} \rightarrow 0$, we obtain

$$
\left\langle g, d * d^{*}\right\rangle=\lim \left\langle g, d_{n} * d_{n}^{*}\right\rangle \geq 0
$$

as $g$ is positive definite on $A$. So we have shown that $g * h$ is positive definite on $G$.

We thus have finished the proof of Proposition 1.15. This also marks the end of the proof of Theorem 1.6.

Theorem 1.16. The $a x+b$-group does not have property $(*)_{p}$ for any $p, 0<$ $p<\infty$.

Remark. Since $(*)_{p}$ implies $(*)_{2 p}$ it suffices to disprove $(*)_{p}$ for large values of $p$.

Let $G$ be the $a x+b$-group, i.e. $G=\{(a, b) \mid a>0, b \in R\}$ with $(a, b)(c, d)=$ $(a c, b+a d)$ with its usual topology. We have $e=(1,0),(a, b)^{-1}=\left(a^{-1},-\frac{b}{a}\right)$ and $(c, d)^{-1}(a, b)(c, d)=\left(a,-\frac{d}{c}+c^{-1}(b+a d)\right)$. Then $a^{-2} d a d b$ is a left Haar measure and $\Delta(a, b)=a^{-1}$ the corresponding modular function. Let $H=$ $\left\{\left(e^{n}, 0\right) \mid n \in Z\right\}, 0<\eta<\frac{1}{8}$ and $V=\left\{\left(e^{r}, s\right) \mid r, s \in(-\eta, \eta)\right\}$. For $x \neq y \in H$ the sets $V x V^{-1}$ and $V y V^{-1}$ are disjoint, as the first coordinates of their points are in $\left\{e^{z}|| z-\log x_{1} \mid<\frac{1}{4}\right\}$ and $\left\{e^{z}|| z-\log y_{1} \mid<\frac{1}{4}\right\}$ respectively and $\left|\log x_{1}-\log y_{1}\right| \geq$ 1 . Let $\chi \xi$ be the characteristic function of $\left(\xi^{-1}, \xi\right) \subset\left(e^{-\eta}, e^{\eta}\right)$ and $f \in L^{2}(R)^{+}$ vanish outside $(-\eta, \eta)$. Then $h=\chi \xi \otimes f$ vanishes outside $V$.

Lemma 1.17. $g=\sum_{x \in H} h * \delta_{x} * \breve{h} \cdot \Delta(x)^{1 / 2}$ is a continuous positive definite function on $G$. 
Proof. (i) Each summand is continuous, the sets of points where the summands are nonzero are pairwise disjoint (see above) and every compact set is met by a finite number of these sets only, so the sum is continuous.

$$
\text { (ii) Let } \begin{aligned}
k \in K(G) . \text { Then }\left\langle g, k^{*} * k\right\rangle & =\sum_{x \in H}\left\langle h * \delta_{x} * \check{h} \cdot \Delta(x)^{1 / 2}, k^{*} * k\right\rangle \\
& =\sum_{x \in H}\left\langle\bar{k} * h * \delta_{x} \cdot \Delta(x)^{1 / 2}, k * h\right\rangle
\end{aligned}
$$

As the support of $k * h$ and hence of $\bar{k} * h=\overline{k * h}$ is compact, only finitely many summands are nonzero. Viewed as a function of $x$, the general term of the above sum is a coefficient of a unitary antirepresentation of $H$, hence positive definite. So the sum is $\geq 0$ (and hence $g$ is positive definite) by the following wellknown Lemma. (Not having found a reference, we include a proof).

Lemma 1.18. For $H$ amenable and unimodular and $p$ positive definite and integrable on $H$, we have $\int_{H} p \geq 0$.

Proof. Let $f_{n} \in K(H)$ be such that $f_{n} * \tilde{f}_{n} \rightarrow 1$ uniformly on compact sets. As $\tilde{f}_{n}=f_{n}^{*}$, we have $\int_{H} p=\lim \int_{H}\left(f_{n} * f_{n}^{*}\right) \cdot p \geq 0$.

Fix the neighbourhood $U=\left(e^{-\frac{1}{2}}, e^{\frac{1}{2}}\right) \times(-1,1)$ of the identity in $G$. We want to compare $\int_{U}|g|^{p}=\int_{U}|h * \check{h}|^{p}$ with $\int_{x U}|g|^{p}=\int_{U}|x g|^{p}=\int_{U} \mid \delta_{x^{-1}} * h * \delta_{x} * \breve{h}$. $\left.\Delta(x)^{\frac{1}{2}}\right|^{p}$.

Writing down the intergrals for $h * \check{h}$ and $\left(\delta_{x-1} * h * \delta_{x} * \check{h}\right) \chi_{U}$ in both cases yields a product of $\int \chi_{\xi}\left(z_{1} y_{1}\right) \chi_{\xi}\left(y_{1}\right) y_{1}^{-2} d y_{1}$ times a "perturbed" convolution on $R$ (with a possible cutoff by $\chi_{U}$ in the second case), the perturbation coming from the group multiplication. If we choose $\xi>1$ sufficiently near to one, the effect of this perturbation can be made as small as we please in \|\|$_{p}$ - norm (supposing $f \in L^{p}(R)$ above). So we may assume that (except for the convolution with the Dirac measures) the convolution in the second coordinate is just ordinary convolution on $R$. This implies that in the ratio $\int_{U}|x g|^{p} / \int_{U}|g|^{p}$ the contribution of the first coordinate cancels out, so the problem is reduced to one dimension. We first determine what $\Delta(x)^{\frac{1}{2}} \delta_{x^{-1}} * h * \delta_{x}$ looks like in the second coordinate: For $x=\left(e^{n}, 0\right) \in H$ we have $\Delta(x)^{\frac{1}{2}}\left(\delta_{x-1} * h * \delta_{x}\right)\left(y_{1}, y_{2}\right)=$ $e^{-\frac{n}{2}} h\left(\left(e^{n}, 0\right)\left(y_{1}, y_{2}\right)\left(e^{-n}, 0\right)\right) e^{n}=e^{\frac{n}{2}} h\left(y_{1}, e^{n} y_{2}\right)=\chi_{\xi}\left(y_{1}\right) \cdot e^{\frac{n}{2}} f\left(e^{n} y_{2}\right)$.

For real $c>0$ and $r \in L^{1}(R)$ define $m_{c} r$ by $\left(m_{c} r\right)(y)=\operatorname{cr}(c y)$ for $y \in R$. Then $m_{c}(r * s)(y)=c r * s(c y)=c \int r(c y-z) s(z) \mathrm{d} z=c^{2} \int r(c y-c z) s(c z) \mathrm{d} z=$ $\int m_{c} r(y-z) m_{c} s(z) \mathrm{d} z=\left(m_{c} r * m_{c} s\right)(y)$.

For conjugate indices $p$ and $q$ we have $\int\left|m_{c} r\right|^{p}=c^{p} \int|r(c y)|^{p} \mathrm{~d} y$ $=c^{p-1} \int|r|^{p}$, so $\left\|m_{c} r\right\|_{p}=c^{\frac{1}{q}}\|r\|_{p}$.

Since the range of the second coordinate in $U$ is $(-1,1)$, we now want to estimate the ratio $\int_{-1}^{1}\left|e^{-\frac{n}{2}} m_{e^{n}}(f) * \check{f}\right|^{p} / \int_{-1}^{1}|f * \check{f}|^{p}$ for a special choice of $f$. This needs some preparation. 
For $f(y)=y^{\alpha} \cdot \chi_{(0, b)}(y)$ we have $m_{c} f(y)=c \cdot c^{\alpha} \cdot y^{\alpha} \cdot \chi_{\left(0, c^{-1} b\right)}(y)$. For $0<a \leq b$ let $f_{[a]}=f \chi_{(0, a)}$. We have

$$
\begin{aligned}
f * \check{f}_{[a]}(v) & =\int(v+y)^{\alpha} \chi_{(0, b)}(v+y) \cdot y^{\alpha} \chi_{(0, a)}(y) d y \\
& =\int(v+y)^{\alpha} y^{\alpha} \chi_{(-v, b-v) \cap(0, a)}(y) d y \\
& =\left\{\begin{array}{cl}
0 & \text { if } v \leq-a \\
\int_{-v}^{a}\left(v y+y^{2}\right)^{\alpha} d y & \text { if }-a \leq v \leq 0
\end{array}\right.
\end{aligned}
$$

(We do not need the other values. If $a=b$, the function is symmetric).

For $-a \leq v<0$ we obtain

$$
\begin{aligned}
f * \breve{f}_{[a]}(v) & =\int_{-v}^{a}\left(y^{2}+v y+\left(\frac{v}{2}\right)^{2}-\left(\frac{v}{2}\right)^{2}\right)^{\alpha} d y \\
& =\int_{-v}^{a}\left(\left(y+\frac{v}{2}\right)^{2}-\left(\frac{v}{2}\right)^{2}\right)^{\alpha} d y \\
& =\left(\frac{v}{2}\right)^{2 \alpha} \int_{-v}^{a}\left[\left(\frac{y}{v / 2}+1\right)^{2}-1\right]^{\alpha} d y
\end{aligned}
$$

and, letting $\frac{y}{v / 2}+1=r$, hence $y=\frac{v}{2}(r-1)$,

$$
\begin{aligned}
& =\left(\frac{v}{2}\right)^{2 \alpha} \int_{-1}^{\frac{a}{v / 2}+1}\left(r^{2}-1\right)^{\alpha} \cdot \frac{v}{2} d r \\
& =-\left(\frac{v}{2}\right)^{2 \alpha+1} \int_{1}^{-\left(\frac{a}{v / 2}+1\right)}\left(r^{2}-1\right)^{\alpha} d r
\end{aligned}
$$

now letting $r=\cosh t$

$$
=-\left(\frac{v}{2}\right)^{2 \alpha+1} \int_{0}^{\operatorname{arccosh}\left(-\frac{a}{v / 2}-1\right)}(\sinh t)^{2 \alpha} \sinh t d t
$$

For $a=b$, taking into account the symmetry of $f * \check{f}$, we get

$$
\|f * \check{f}\|_{p}^{p}=2 \cdot \int_{0}^{b}\left(\frac{v}{2}\right)^{(2 \alpha+1) p}\left(\int_{0}^{\operatorname{arccosh}\left(\frac{b}{v / 2}-1\right)}(\sinh t)^{(2 \alpha+1)} d t\right)^{p} d v
$$


and letting $v=b s$

$$
\begin{aligned}
& =\operatorname{const} b^{(2 \alpha+1) p} \cdot b \int_{0}^{1} s^{(2 \alpha+1) p}\left(\int_{0}^{\operatorname{arccosh}\left(\frac{2}{s}-1\right)}(\sinh t)^{(2 \alpha+1)} d t\right)^{p} d s \\
& \leq \text { const } b^{(2 \alpha+1) p+1} \int_{0}^{1} s^{(2 \alpha+1) p}\left(\left[\frac{1}{2 \alpha+1} e^{(2 \alpha+1) t}\right]_{0}^{\log \left(\frac{4}{s}-2\right)}\right)^{p} d s
\end{aligned}
$$

(as $\operatorname{arccosh} y \leq \log 2 y$ )

$$
\leq \text { const } b^{(2 \alpha+1) p+1} \int_{0}^{1} s^{(2 \alpha+1) p}\left(\frac{\left(\frac{4}{s}-2\right)^{2 \alpha+1}-1}{2 \alpha+1}\right)^{p} d s
$$

As the integral is finite if $(2 \alpha+1) p>-1$ (and $\alpha \neq-\frac{1}{2}$ ), in this case we have $\|f * \check{f}\|_{p}^{p} \leq$ const $b^{(2 \alpha+1) p+1}$ where the constant depends on $\alpha$ and $p$ only.

For $0<a<b$ we obtain

$$
\left\|f * \check{f}_{[a]}\right\|_{p}^{p} \geq \int_{-a}^{0}\left(-\left(\frac{v}{2}\right)^{2 \alpha+1}\right)^{p}\left(\int_{0}^{\operatorname{arccosh}\left(\frac{2 a}{-v}-1\right)}(\sinh t)^{2 \alpha+1} d t\right)^{p} d v
$$

and letting $v=-$ as

$$
\begin{aligned}
& =a^{(2 \alpha+1) p} \cdot a \int_{0}^{1}\left(\frac{s}{2}\right)^{(2 \alpha+1) p}\left(\int_{0}^{\operatorname{arccosh}\left(\frac{2}{s}-1\right)}(\sinh t)^{2 \alpha+1} d t\right)^{p} d s \\
& =\text { const } \cdot a^{(2 \alpha+1) p+1}, \text { the constant depending on } \alpha \text { and } p \text { only }
\end{aligned}
$$

We now come back to our problem of comparing \|\|$_{p}$ - norms. If $b<\eta$, the support of $f$ is contained in $(-\eta, \eta)$. Now consider the "compressed" function $F=m_{c} f$ for large $c>0$ (in fact $c$ will be of the form $e^{n}$ ). If, in the construction of $g$, we replace $f$ by $F$, then for $x=(c, 0)$ the estimation of the ratio of the $p$-norms of $x g$ and $g$ on $U$ amounts to estimating the ratio of the $p$-norms of $c^{\frac{1}{2}} m_{c^{-1}}(F) * \check{F}$ and $F * \check{F}$ (note that both functions are supported in $(-1,1)$, the second coordinate range of $U)$. We have

$$
\begin{gathered}
\|F * \check{F}\|_{p}=\| m_{c}(f) * m_{c}\left(f \check{)}\left\|_{p}=\right\| m_{c}(f) * m_{c}(\check{f})\left\|_{p}=\right\| m_{c}(f * \check{f}) \|_{p}\right. \\
=c^{\frac{1}{q}}\|f * \check{f}\|_{p} \text { and } \\
\left\|c^{\frac{1}{2}} m_{c^{-1}} F * \check{F}\right\|_{p}=c^{\frac{1}{2}}\left\|f * m_{c}(f)\right\|_{p}=c^{\frac{1}{2}} c c^{\alpha}\left\|f * \check{f}_{\left[c^{-1} b\right]}\right\|_{p} \\
\geq c^{\frac{1}{2}+1+\alpha} \cdot \mathrm{const} \cdot\left(c^{-1} b\right)^{2 \alpha+1+\frac{1}{p}} \\
\geq \text { const } \cdot c^{\frac{1}{2}+1+\alpha-2 \alpha-1-\frac{1}{p}}\|f * \check{f}\|_{p} .
\end{gathered}
$$


The quotient is $\geq c^{\frac{1}{2}-\alpha-\frac{1}{p}-\frac{1}{q}}$. If $\alpha=-\frac{1}{2}-\frac{1}{4 p}$, the quotient is $\geq c^{\frac{1}{4 p}}$ which tends to infinity if $c \rightarrow \infty$. Note that $(2 \alpha+1) p=\left(-1-\frac{1}{2 p}+1\right) p=-\frac{1}{2}>-1$ as was required above. For $p \geq 2$ we do not have $f \in L^{p}$ or even $f \in L^{2}$ for such $\alpha$, and the first requirement was essential, but this can be arranged by replacing $f$ by $f \cdot \chi(\varepsilon, b)$ where $\varepsilon>0$ is sufficiently small. As we let $\varepsilon \rightarrow 0$, the norms for the changed function tend to the respective norms for the original function $f$. So $\mathrm{G}$ does not have property $(*)_{p}$ for large $p$ and hence not for any $p \in(0, \infty)$.

Acknowledgements. I am grateful to John Fournier for drawing my attention to this problem, to him and to Michael Cowling for fruitful discussions, and to Karl Heinrich Hofmann for immediately and more than completely answering a structure theoretic question of mine.

\section{References}

[Ba] Bachelis, G.F.: On the upper and lower majorant properties in $L^{p} \mathrm{G}$ ), Quart. J. Math. Oxford 24, 119-128 (1973).

[Be Da Du] Bertrandias, J.P., Datry, C., Dupuis C.: Unions et intersections d'espaces $L^{\mathrm{P}}$ invariantes par translation et convolution, Ann. Inst. Fourier 28, 53-84 (1978).

[Di] Dixmier, J.: C*-Algebras, North Holland Publishing Company 1977.

[Fd] Folland, G.B.: A course in abstract Harmonic Analysis, CRC Press 1995

[Fo 1] Fournier, J.J.F.: Majorants and $L^{P}$ norms, Israel J. 18, 157-166 (1974).

[Fo 2] Fournier, J.J.F.: Local and global properties of functions and their Fourier transforms, Tôhoku Math. J. 49, 115-131 (1997).

[Gr Mz] Grosser, S., Moskowitz, M.: Compactness conditions in topological groups, J. Reine Angew. Math. 246, 1-40 (1971).

[He Ro] Hewitt, E., Ross, K.A.: Abstract Harmonic Analysis, Vol. I, Springer Verlag 1963.

[Ho Mo] Hofmann, K.H., Mostert, P.S.: Splitting in topological groups, Memoirs Amer. Math. Soc. 43 (1963).

[Ka On Ta] Kawazoe, T., Onoe, Y., Tachizawa, K.: Functions on the real line with nonnegative Fourier transforms, Tôhoku Math J. 46, 311-320 (1994).

[Mo] Mostert, P.S.: Local cross sections in locally compact groups, Proc. Amer. Math. Soc. 4, 645-649 (1953).

[Ra] Rains, M.: On functions with nonnegative Fourier transforms, Indian J. Math. 27, 41-48 (1985).

[Sh] Shapiro, H.S.: Majorant problems for Fourier coefficients, Quart.J. Math. Oxford 26, 9-18 (1975).

[Wa] Wainger, S.: A problem of Wiener and the failure of a principle for Fourier series with positive coefficients, Proc. Amer. Math. Soc. 20, 16-18 (1969).

[Ze] Zelmanov, E.I.: On periodic compact groups, Israel J. 77, 83-95 (1982). 